\title{
Controversies in reproductive technologies: Diagnostic testing for infectious agents
}

\author{
Joanne Embree MD FRCPC
}

P roblems related to the unregulated nature of clinics and laboratories involved in provision of reproductive technologies have been the subject of a number of news reports of the past few years. These reports have been a public relations nightmare for those involved in trying to help couples with fertility problems. Couples, who are presently involved in this process or who had attended some of these clinics in the past, were notified this summer (1999) through press reports that donor sperm have been quarantined and that there is a remote possibility that they may have been exposed to one or more potentially health-threatening infections. The quarantine has also resulted in a disruption in services for many couples.

As new and improved diagnostic tests become available, many have become incorporated into screening protocols to prevent the transmission of infection to the recipients of donor ova and sperm. There is always a delay among the development of a diagnostic test, the determination of its effectiveness in improving the diagnosis of infection, establishing its usefulness as a screening tool and, finally, a formal recommendation for its routine use by regulating bodies. Once a screening test for infection has been recommended in a donation program, it is usually introduced universally into the donor screening protocols in the public sector, which is responsible for blood and organ donations. However, this may not have been the case in the private sec- tor clinics involved in reproductive technologies, which have not been as closely regulated. Hence, there was the recent publicity about this issue, the incorporation of previously written practice guidelines as formal requirements for testing and the quarantine of donor specimens from many clinics.

There are a number of unresolved issues related to the testing for infectious diseases in this situation. One involves the utilization of newer polymerase chain reaction and other DNA replicating technologies for semen or urine samples as a means of optimal follow-up testing of sperm donors for the classic sexually transmitted diseases, chlamydia and gonorrhea. These tests were not mentioned in the guidelines developed in 1996. The use of these tests could replace the requirement for obtaining repeated urethral swab specimens, a somewhat unpopular procedure. The men involved in the donor programs, in theory, are an extremely low risk population for these infections. The new technologies are likely sufficiently sensitive to detect the rare infection, should it occur. However, policy makers do not have the results of a study specifically designed to determine this, and it is unlikely that they ever will in view of the numbers needed to conduct such a study. However, in this instance, there is good evidence from studies completed in other populations to justify their use.

Another murky area involves how to deal with the results of testing for infections, such as ureaplasma, 
mycoplasma and cytomegalovirus (CMV). Given that these infections are extremely common in the general population, what increased risk to recipient couples do donors with evidence of these chronic infections actually pose? The answer to this question is not known. Screening and the elimination of donors with evidence of these infections from the donor pool significantly decrease the number of donors available for these programs.

Ureaplasma infection has been epidemiologically linked to both infertility and prematurity. A causative role for this organism has not yet been clearly established, despite a great deal of effort to do so. Epidemiological links with mycoplasma, and infertility and prematurity are less clear. Therefore, policy makers need to consider carefully the true need for including tests for these organisms as part of the screening protocols. If the decision is made that tests should be done for ureaplasma and/or mycoplasma, this should likely be to specifically match uninfected recipient couples with uninfected donors. The purpose would be to ensure that uninfected couples were not exposed to these organisms in the programs.

If a woman acquires primary CMV infection during pregnancy, her infant is at high risk of congenital CMV infection. Hence, there is concern about transmission of this particular infection at the time of conception. The detection of antibodies to CMV does not necessarily imply its presence in semen. This situation is actually quite rare unless the individual has recently been infected, has unprotected sex with multiple partners or is immune suppressed due to HIV infection. Testing semen for the presence of CMV, with exclusion of samples testing positive, would seem to be a better solution for this problem. This should be possible with the recent development of polymerase chain reaction technology for detection of CMV. Testing could be incorporated into protocols using this type of technology for detection of chlamydia and gonorrhea. Alternatively, matching CMV recipient couples with seronegative donors may be a consideration for handling this infection as well. However, these increased requirements will increase the complexity of the matching process.

One final issue that follows from the introduction of changes in the recommended screening protocols is what to do about testing the previous donors and/or recipients. These individuals did not have the benefit of the new screening protocols and, therefore, may be at risk. What procedures should be put into place? Who should be called back for testing? How should this be done? The answers likely depend on what test is being introduced. Would the introduction of a new test for gonorrhea or chlamydia necessarily result in the need for a callback of previous donors and recipient couples? One would suspect that this would not be generally necessary because of the rarity of these infections among donors and the quality of the previous screening, provided that it has taken place. The high background prevalence of CMV or ureaplasma infection among the general population, coupled with no need to treat iden- tified healthy carriers, seem to make notification and further screening of previous clinic participants unnecessary. The introduction of HIV testing obviously should have been coupled with a callback of previous donors for testing and a warning to previous recipients that they should consider testing if the donors could not be located and their infection status needs to be clarified. This was a newly introduced infection in the community; therefore, the establishment of a risk period dating from the time that the infection was likely introduced into the population was a reasonable precaution. The recent introduction of testing for hepatitis $\mathrm{C}$, human $\mathrm{T}$ cell lymphotrophic virus type 1 and HIV-2 infection should be handled in a similar fashion, despite an exceptionally low risk of transmission of any of these infections in this situation. Both human T cell lymphotrophic virus type 1 and HIV-2 are rare, and the sexual nature of transmission of hepatitis $\mathrm{C}$ is questionable. However, these infections are important for infected individuals to know about because there are methods to prevent further transmission and specific treatments are being developed.

On occasion, the callback of donors for testing may be problematic if they are no longer with the program or the program has closed. They either cannot be located or may refrain from being tested. In this situation, recipients need to be contacted and notified of the situation. Again, there may be difficulties in locating these individuals. This process is stressful for parents, who went through so much to conceive their children, despite the almost negligible risk that they or their children have acquired one of these infections. For those who did not have a successful pregnancy, this process reopens very painful wounds. For the clinics involved, there are concerns of legal liabilities. There should be no legal liability for not performing nonexistent tests or for following the accepted standards of care at the time. However, there is, at least, a moral responsibility to provide former patients with the relevant information that they need concerning new developments. The introduction and modification of testing procedures will continue to occur. Thus, similar situations will continue to occur. One practical way to minimize the need for continued callback of former donors or patients is to save sufficient quantities of serum and blood spots, along with ova and sperm, for future testing. This will involve a relatively small expense relative to the high cost of the callback process.

Clearly, closer regulation of the provision of reproductive technologies is warranted. Regulating bodies developing protocols for infectious disease screening must ensure that the protocols are appropriate. They also need to take into account that newer technologies and procedures will be developed and to make appropriate provisions for this occurrence. Finally, infectious disease specialists, with expertise in the area of sexually transmitted organisms, should be formally involved in the generation and review of these protocols. 